\title{
Focusing Modeling of OPFC Linear Array Transducer by Using Distributed Point Source Method
}

\author{
Ziping Wang and Ying Luo \\ Faculty of Civil Engineering and Mechanics, Jiangsu University, Zhenjiang, Jiangsu 212013, China \\ Correspondence should be addressed to Ziping Wang; wzpxx2004@126.com
}

Received 28 January 2014; Accepted 22 February 2014; Published 16 April 2014

Academic Editor: Baojian Hong

Copyright ( 2014 Z. Wang and Y. Luo. This is an open access article distributed under the Creative Commons Attribution License, which permits unrestricted use, distribution, and reproduction in any medium, provided the original work is properly cited.

\begin{abstract}
The improvement of ultrasonic phased array detection technology is a major concern of engineering community. Orthotropic piezoelectric fiber composite (OPFC) can be constructed to multielement linear array which may be applied conveniently to actuators and sensors. The phased array transducers can generate special directional strong actuator power and high sensitivity for its orthotropic performance. Focusing beam of the linear phased array transducer is obtained simply only by adjusting a parabolic time delay. In this work, the distributed point source method (DPSM) is used to model the ultrasonic field. DPSM is a newly developed mesh-free numerical technique that has been developed for solving a variety of engineering problems. This work gives the basic theory of this method and solves the problems from the application of new OPFC phased array transducer. Compared with traditional transducer, the interaction effect of two OPFC linear phased array transducers is also modeled in the same medium, which shows that the pressure beam produced by the new transducer is narrower or more collimated than that produced by the conventional transducer at different angles. DPSM can be used to analyze and optimally design the OPFC linear phased array transducer.
\end{abstract}

\section{Introduction}

The ultrasonic phased array detection technology which was proposed by Crawford of America Conoco Company originated in the 1960s [1]. The phased array transducer is formed by connecting different components and space distribution of piezoelectric elements. The structure can be detected with rapid multi-angles by controlling emitting order and time delay. These arrays have been also utilized in the field of nondestructive evaluation (NDE) of materials $[2,3]$. But conventional phased array transducers have some deficiencies such as brittle fracture, different design ability, and illegitimate mechanical-electrical parameters [4] with piezoelectric material as core element, and the final resolution will be affected. Therefore, the ultrasonic phased array detecting technology can be further improved by improving the quality of key materials and developing new phased array transducer. Compared with traditional piezoceramics, orthotropic piezoelectric composite materials (OPFC) which are used as actuator/sensor elements in damage detection for engineering structures show the obvious advantages for their excellent orthotropic characteristic $[5,6]$.

The group of Luo [7] has developed models of actuators and sensors with OPFC to satisfy the detection of different structures. These models of sensors will be used to fabricate new phased array transducers in many different engineering structures inspection. OPFC phased array is treated as a linear array consisting of single element sources. The new structure has orthotropic performance. This special performance can be used to actuate and receive the stress wave in different directions and reduce the interfering signals.

In this paper, new OPFC phased array transducers are semianalytically modeled by the distributed point source method (DPSM). The DPSM is used to model ultrasonic transducers for computing pressure and velocity fields generated by the new transducers. The group of Placko [8] first developed the new method for ultrasonic transducer simulation. In the present paper, an OPFC phased array transducer is modeled using DPSM to obtain the ultrasonic field in a homogeneous medium. The results compare the 
phased array transducer and conventional planar transducer generated ultrasonic beam in different directions. The importance of the interaction effect between two phased arrays is also considered by computing the ultrasonic field.

\section{DPSM Theory Analysis}

Based on a spatial distribution of point source, DPSM modeling can be used to compute the pressure field generated by an ultrasonic transducer which is placed in homogeneous or nonhomogeneous fluid media. A traditional transducer composed of many point sources which oscillate at the same time. Equation (1) shows the total field at a point generated by $N$ number of point sources, but the point sources are excited at different times for a phased array transducer. Let the $m$ th point source of strength $A_{m}$ be excited at time $t_{m}$; the pressure at the distance $r_{m}$ from a specific point source is given in (2):

$$
\begin{gathered}
p(x)=\sum_{m=1}^{N} p_{m}\left(r_{m}\right)=\sum_{m=1}^{N} A_{m} \frac{\exp \left(i k_{f} r_{m}\right)}{r_{m}} \\
p_{m}(r)=A_{m} \frac{\exp \left(i k_{f} r_{m}-i \omega t_{m}\right)}{r_{m}},
\end{gathered}
$$

where $\omega$ is the angular frequency and $k_{f}$ is the wave number. For the phased array transducer, the point sources on the surface are assumed to be excited after different time intervals $V t_{m}$ and $t_{m}$ can be expressed as

$$
t_{m}=t-V t_{m}
$$

where $t$ is a reference time $t$. If there are $N$ point sources distributed over the element surface, as shown in Figure 1, the total pressure at point $x$ can be obtained:

$$
p(x)=\sum_{m=1}^{N} p_{m}\left(r_{m}\right)=\sum_{m=1}^{N} A_{m} \frac{\exp \left(i k_{f} r_{m}-i \omega\left(t-V t_{m}\right)\right)}{r_{m}}
$$

where $r_{m}$ is the distance of the $m$ th point source from $x$. From the pressure-velocity relation [9], the velocity in $r$ direction which is generated by the $m$ th point source is given by

$$
\begin{aligned}
v_{m}(r) & =\frac{A_{m}}{i \omega \rho} \frac{\partial}{\partial r}\left(\frac{\exp \left(i k_{f} r-i \omega\left(t-V t_{m}\right)\right)}{r}\right) \\
& =\frac{A_{m}}{i \omega \rho}\left(\frac{i k_{f} \exp \left(i k_{f} r\right)}{r}-\frac{\exp \left(i k_{f} r\right)}{r^{2}}\right)
\end{aligned}
$$

$$
\begin{aligned}
& \times \exp \left(-i \omega\left(t-V t_{m}\right)\right) \\
= & \frac{A_{m}}{i \omega \rho} \frac{\exp \left(i k_{f} r\right)}{r}\left(i k_{f}-\frac{1}{r}\right) \exp \left(-i \omega\left(t-V t_{m}\right)\right) .
\end{aligned}
$$

After adding the contributions of $N$ sources, the velocity components in directions $x_{1}, x_{2}$, and $x_{3}$ (see Figure 1) are obtained:

$$
\begin{aligned}
v_{1}(x)=\sum_{m=1}^{N} v_{1 m}\left(r_{m}\right)= & \sum_{m=1}^{N} \frac{A_{m}}{i \omega \rho} \frac{x_{1 m} \exp \left(i k_{f} r_{m}\right)}{r_{m}^{2}} \\
& \times\left(i k_{f}-\frac{1}{r}\right) \exp \left(-i \omega\left(t-V t_{m}\right)\right) \\
v_{2}(x)=\sum_{m=1}^{N} v_{2 m}\left(r_{m}\right)= & \sum_{m=1}^{N} \frac{A_{m}}{i \omega \rho} \frac{x_{2 m} \exp \left(i k_{f} r_{m}\right)}{r_{m}^{2}} \\
& \times\left(i k_{f}-\frac{1}{r}\right) \exp \left(-i \omega\left(t-V t_{m}\right)\right) \\
v_{3}(x)=\sum_{m=1}^{N} v_{3 m}\left(r_{m}\right)= & \sum_{m=1}^{N} \frac{A_{m}}{i \omega \rho} \frac{x_{3 m} \exp \left(i k_{f} r_{m}\right)}{r_{m}^{2}} \\
& \times\left(i k_{f}-\frac{1}{r}\right) \exp \left(-i \omega\left(t-V t_{m}\right)\right) .
\end{aligned}
$$

Because the transducer surface vibrates with a nonzero velocity in $x_{3}$ direction only, on the surface, $v_{1}=0$ and $v_{2}=0$. For traditional transducers, $v_{3}=v_{0}$ and for phased array transducers, $v_{3}=v_{0} \exp \left[-i \omega\left(t-V t_{m}\right)\right]$. If the observation point $x$ is placed on the surface of transducer, the three expressions of equation (6) should be equal to 0,0 , and $v_{0} \exp \left[-i \omega\left(t-V t_{m}\right)\right]$, respectively. Thus, $3 N$ equations will be obtained from $N$ points on the transducer surface. We get more equations than unknowns. To get the same number of unknowns as $3 N$ equations, the number of unknowns can be increased from $N$ to $3 N$ by replacing each point source by a triplet source. All sources are placed on the same plane at $x_{3}=-r_{s}$ parallel to the transducer surface. Random orientations of triplet sources are necessary to preserve the isotropic material properties. By solving $3 \mathrm{~N}$ linear equations, the source strength $A_{m}$ of all point sources can be obtained. After getting $A_{m}$, the pressure $p(x)$ can be calculated at any point from (1). The velocity components parallel to the transducer face are not necessarily continuous because slippage may occur between transducer face and the fluid. Equation (6) can be combined into the following matrix equation:

$$
V_{S}=M_{S S} A_{S}
$$

where $V_{S}$ is the $(3 N \times 1)$ vector of the velocity components at $N$ number of surface points $x . A_{S}$ is the $(3 N \times 1)$ vector of the strengths of $3 N$ number of point sources. $M_{S S}$ is the 


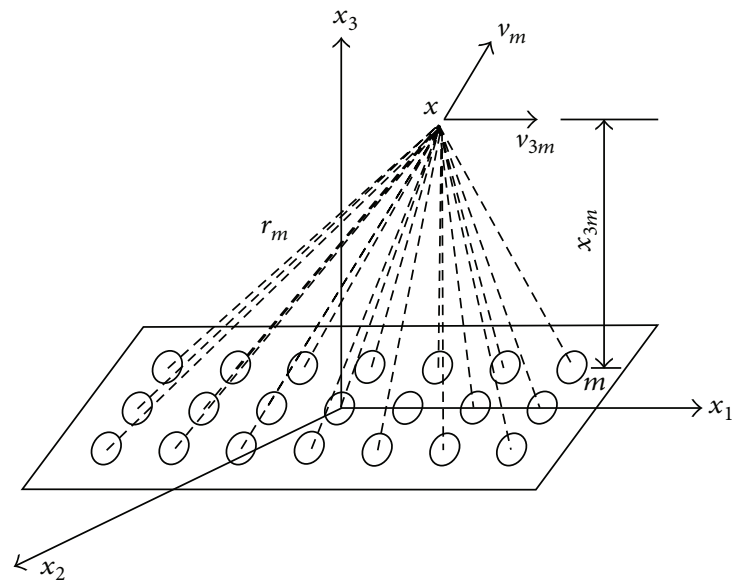

FIGURE 1: Geometric model of DPSM.

$(3 N \times 3 N)$ matrix relating the two vectors $V_{S}$ and $A_{S}$. So $V_{S}$ can be written as

$$
\left\{V_{S}\right\}^{T}=\left[\begin{array}{llllllllll}
v_{1}^{1} & v_{2}^{1} & v_{3}^{1} & v_{1}^{2} & v_{2}^{2} & v_{3}^{2} & \cdots & v_{1}^{N} & v_{2}^{N} & v_{3}^{N}
\end{array}\right] .
$$

Vector $A_{s}$ of the source strengths is given by

$$
\left\{A_{S}\right\}^{T}=\left[\begin{array}{llllllllll}
A_{1} & A_{2} & A_{3} & A_{4} & A_{5} & A_{6} & \cdots & A_{(3 N-2)} & A_{(3 N-1)} & A_{3 N}
\end{array}\right] .
$$

$$
M_{S S}=\left[\begin{array}{ccccccc}
f\left(x_{11}^{1}, r_{1}^{1}\right) & f\left(x_{12}^{1}, r_{2}^{1}\right) & f\left(x_{13}^{1}, r_{3}^{1}\right) & \ldots & \cdots & f\left(x_{1(3 N-1)}^{1}, r_{3 N-1}^{1}\right) & f\left(x_{1(3 N)}^{1}, r_{3 N}^{1}\right) \\
f\left(x_{21}^{1}, r_{1}^{1}\right) & f\left(x_{22}^{1}, r_{2}^{1}\right) & f\left(x_{23}^{1}, r_{3}^{1}\right) & \ldots & \ldots & f\left(x_{2(3 N-1)}^{1}, r_{3 N-1}^{1}\right) & f\left(x_{2(3 N)}^{1}, r_{3 N}^{1}\right) \\
f\left(x_{31}^{1}, r_{1}^{1}\right) & f\left(x_{32}^{1}, r_{2}^{1}\right) & f\left(x_{33}^{1}, r_{3}^{1}\right) & \ldots & \ldots & f\left(x_{3(3 N-1)}^{1}, r_{3 N-1}^{1}\right) & f\left(x_{3(3 N)}^{1}, r_{3 N}^{1}\right) \\
\cdots & \cdots & \cdots & \cdots & \ldots & \cdots & \cdots \\
f\left(x_{31}^{N}, r_{1}^{N}\right) & f\left(x_{32}^{N}, r_{2}^{N}\right) & f\left(x_{33}^{N}, r_{3}^{N}\right) & & & f\left(x_{3(3 N-1)}^{N}, r_{3 N-1}^{N}\right) & f\left(x_{3(3 N)}^{N}, r_{3 N}^{N}\right)
\end{array}\right],
$$

where

$$
\begin{aligned}
f\left(x_{j m}^{n}, r_{m}^{n}\right)= & \frac{x_{j m}^{n} \exp \left(i k_{f} r_{m}^{n}\right)}{i \omega \rho\left(r_{m}^{n}\right)^{2}} \\
& \times\left(i k_{f}-\frac{1}{r_{m}^{n}}\right) \times \exp \left(-i \omega\left(t-V t_{m}^{n}\right)\right) .
\end{aligned}
$$

In the above equations $r_{m}^{n}$ stands for the distance between the $m$ th point source and $n$th aim point of the transducer surface. The first subscript of $j$ of $x$ can take 1,2 , or 3 and indicate the $x_{1}, x_{2}$, or $x_{3}$ directions. The subscript $m$ of $x$ and $r$ can take values from 1 to $3 N$ depending on the considered point source and the superscript $n$ can take values from 1 to $N$. As mentioned formulation from $3 N$ point sources, three boundary conditions of velocity are satisfied to every point of $N$ boundary points. From the point source strengths by inverting matrix $M_{S S}$,

$$
A_{S}=\left[M_{S S}\right]^{-1} V_{S}=N_{S S} V_{S}
$$

Then, the pressure $P_{T}$ and velocity components $V_{T}$, at the observation points, are obtained from the following matrix relation:

$$
\begin{gathered}
P_{T}=Q_{T S} A_{S} \\
V_{T}=M_{T S} A_{S},
\end{gathered}
$$

If the observation points are identical to the transducer surface points, matrix $M_{T S}$ will be the same as $M_{S S}$ of (11). However, by computing the velocity field at different points, $Q_{T S}$ will be as shown 


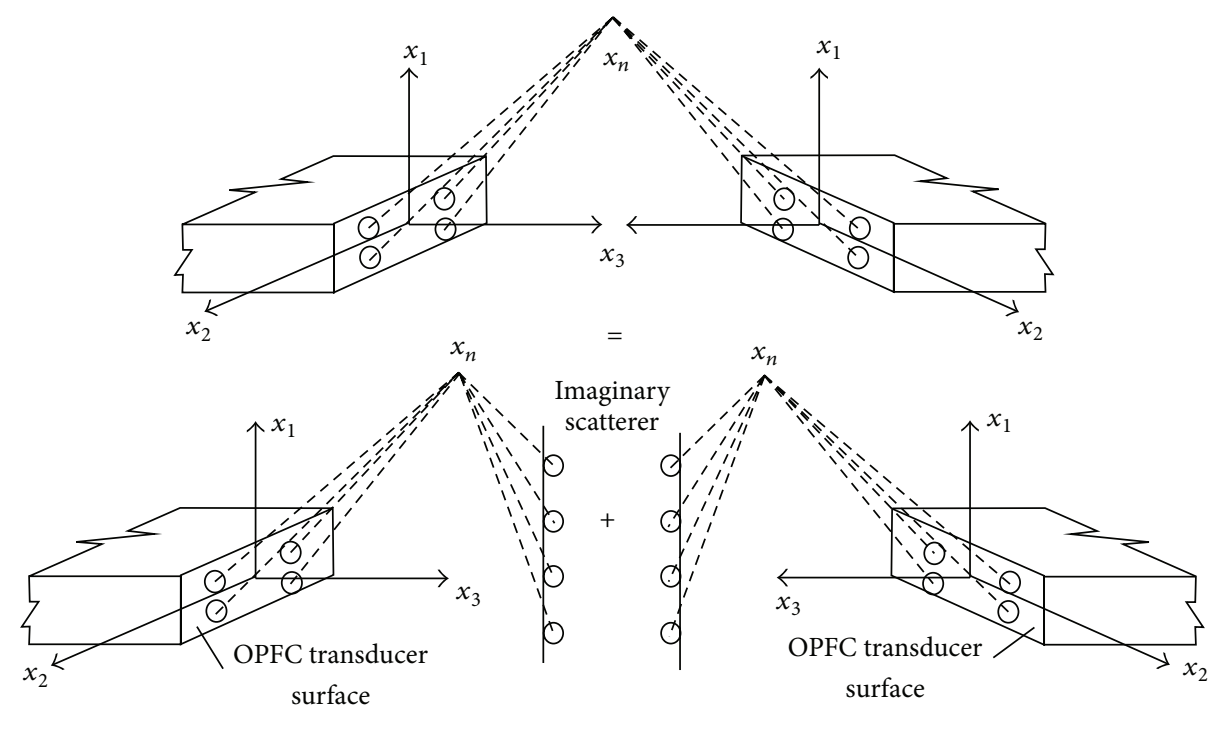

Part 1

Part 2

FigURE 2: The interaction effects of two transducers by superimposing two parts.

$$
Q_{T S}=\left[\begin{array}{ccccc}
\frac{\exp \left(i k_{f} r_{1}^{1}-i \omega\left(t-V t_{1}^{1}\right)\right)}{r_{1}^{1}} & \frac{\exp \left(i k_{f} r_{2}^{1}-i \omega\left(t-V t_{2}^{1}\right)\right)}{r_{2}^{1}} & \cdots & \cdots & \frac{\exp \left(i k_{f} r_{3 N}^{1}-i \omega\left(t-V t_{3 N}^{1}\right)\right.}{r_{3 N}^{1}} \\
\frac{\exp \left(i k_{f} r_{1}^{2}-i \omega\left(t-V t_{1}^{2}\right)\right)}{r_{1}^{2}} & \frac{\exp \left(i k_{f} r_{2}^{2}-i \omega\left(t-V t_{2}^{2}\right)\right)}{r_{2}^{2}} & \cdots & \cdots & \frac{\exp \left(i k_{f} r_{3 N}^{2}-i \omega\left(t-V t_{3 N}^{2}\right)\right.}{r_{3 N}^{2}} \\
\frac{\exp \left(i k_{f} r_{1}^{3}-i \omega\left(t-V t_{1}^{3}\right)\right)}{r_{1}^{3}} & \frac{\exp \left(i k_{f} r_{2}^{3}-i \omega\left(t-V t_{2}^{3}\right)\right)}{r_{2}^{3}} & \cdots & \cdots & \frac{\exp \left(i k_{f} r_{3 N}^{3}-i \omega\left(t-V t_{3 N}^{3}\right)\right)}{r_{3 N}^{3}} \\
\cdots & \cdots & \cdots & \cdots & \cdots \\
\frac{\exp \left(i k_{f} r_{1}^{N_{T}}-i \omega\left(t-V t_{1}^{N_{r}}\right)\right)}{r_{1}^{N_{T}}} & \frac{\exp \left(i k_{f} r_{2}^{N_{T}}-i \omega\left(t-V t_{2}^{N_{r}}\right)\right)}{r_{2}^{N_{T}}} & \cdots & \cdots & \frac{\exp \left(i k_{f} r_{3 N}^{N_{T}}-i \omega\left(t-V t_{3 N}^{N_{r}}\right)\right)}{r_{3 N}^{N_{T}}}
\end{array}\right] .
$$

When two transducers are placed in the same fluid, the pressure field can be modeled by superimposing two simpler parts, as shown in Figure 2. The total field at any point $x_{n}$ can be looked as part 1 and part 2 . The right face is replaced by an imaginary scattering surface in part 1 and the left face is replaced by the imaginary scatterer. The two problems can be modeled as distributed point sources. The total ultrasonic field at point $x_{n}$ generated by the two OPFC phased array transducers can be obtained by combining the above equations:

$$
\begin{aligned}
P_{\text {left }} & =P_{S(\text { left })}^{i}+P_{1 \text { (rightscatterer })}^{i} \\
P_{\text {right }} & =P_{S(\text { right })}^{i}+P_{1(\text { leftscatterer })}^{i} \\
P_{\text {total }} & =P_{\text {left }}+P_{\text {right }},
\end{aligned}
$$

where $P_{\text {left }}$ and $P_{\text {right }}$ are the ultrasonic fields generated by part 1 and part 2, respectively. $P_{\text {total }}$ is the total effect for the two transducers. Note that $P_{1}^{i}$ takes into account the interaction effect. $P_{S}^{i}$ is the pressure field by the original transducer.

\section{The Pressure Field of OPFC Element in a Homogeneous Fluid}

The DPSM theory presented above is used to model ultrasonic field generated in a homogeneous fluid (water) by OPFC ultrasonic phased array transducer. The near field length and the divergence angle can be calculated in the method. Compared with the closed form analytical values, we can validate the accuracy of DPSM. The near field length $N_{F}$ and divergence angle $\phi$ equation can be shown as follows [10]:

$$
\begin{gathered}
N_{F}=\frac{F_{s}}{\pi \lambda} \\
\phi=\arcsin \frac{\lambda}{2 a},
\end{gathered}
$$




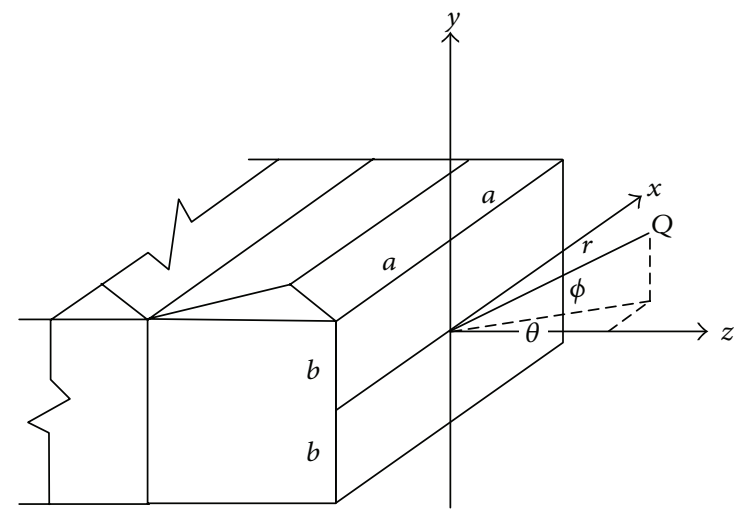

Figure 3: Coordinate coefficient of rectangle source acoustic field of OPFC element.

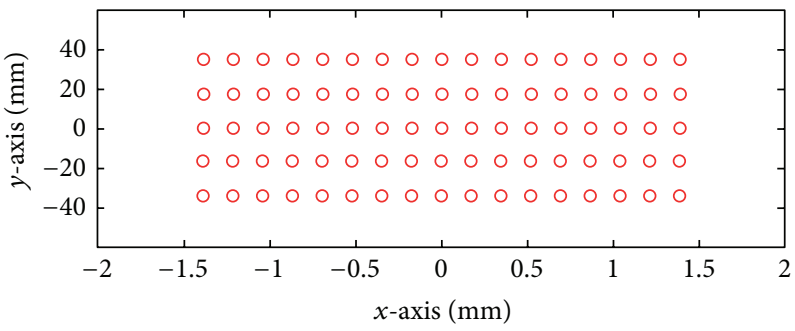

FIGURE 4: Spatial sources distribution.

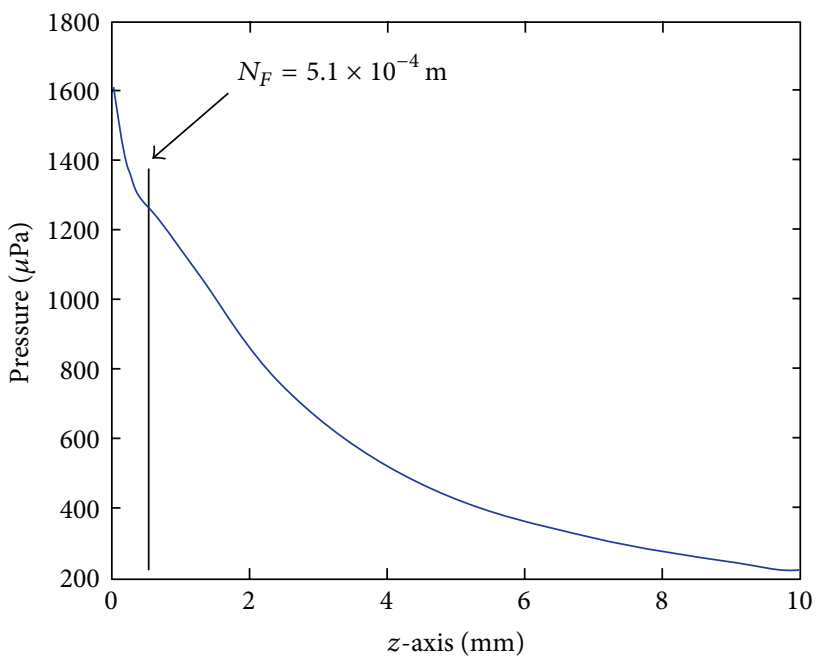

FIgURE 5: Acoustic pressure along $z$-axis.

where $\lambda$ is the wave length, $a$ is the half length of section, and $F_{s}$ is the rectangle area of the transducer, as shown in Figure 3. The analytical expression of the pressure in a homogeneous fluid along the central axis of a transducer is given in (17):

$$
p\left(x_{3}\right)=\frac{P_{0} F_{s}}{\lambda r} .
$$

Figure 4 shows the variations of acoustic pressure along $x_{3}$ axis by DPSM and analytical expression. The signal frequency $f=1 \mathrm{MHz}$. The dimension of the transducer is $21.6 \mathrm{~mm} \times$ $3 \mathrm{~mm} \times 0.8 \mathrm{~mm}$, which is the optimal dimension to design the OPFC element. For the DPSM modeling 80 points are placed at $x_{3}=-r_{s}$, while the transducer surface is at $x_{3}=$ 0 . According to the condition of DPSM geometry problem, $r_{s}=\sqrt{4 a b / 2 \pi N}=\sqrt{(4 \times 0.6) /(2 \pi \times 80)}=0.07 \mathrm{~mm}$. From (16) $N_{F}$ is found to be $0.51 \mathrm{~mm}$. As shown in Figure 3, the theoretical value of $N_{F}$ matches very well with the transition point.

Figure 6 shows the corresponding acoustic pressure variation in $x-z$ plane in DPSM technology. The theoretical divergence angle $\left(\phi=29.8^{\circ}\right)$ is calculated from (17). The measured divergence angle from Figure 5 is about $30^{\circ}$, which 


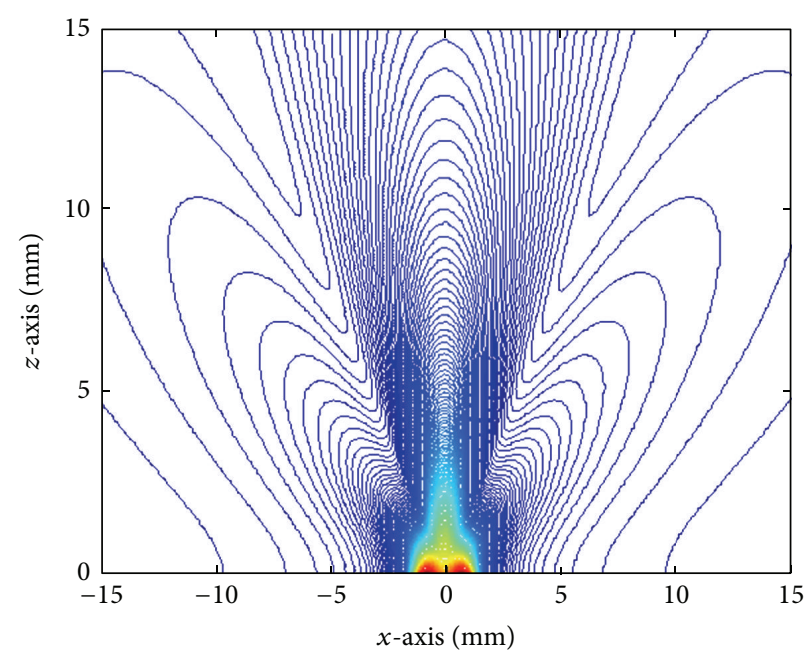

FIGURE 6: Acoustic pressure variation in $x-z$ plane.

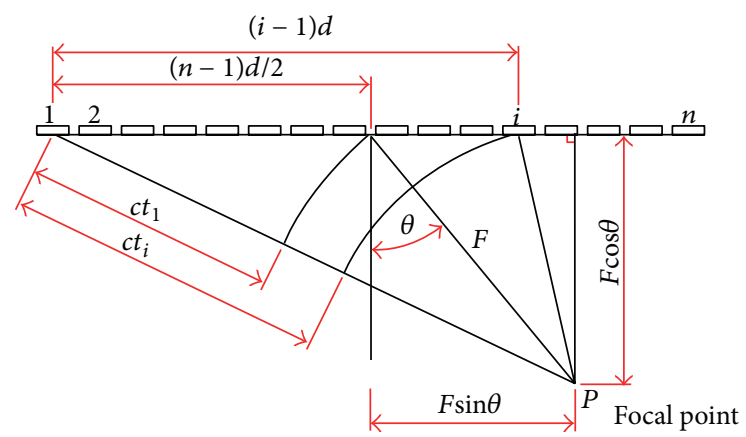

Figure 7: Geometry of linear OPFC phased array for focal point $P$.

is very close to the theoretical value. The two results validate the accuracy of DPSM.

\section{Numerical Simulation Results of OPFC Phased Array Transducer by DPSM}

4.1. Dynamic Focusing and Time Delay Calculation. Focusing beam is accomplished by combining a spherical timing relationship to produce a beam, which is focused at a given range and propagates at a specific angle. The focusing delays can be calculated by the following traditional formula [11]:

$$
t_{n}=\frac{F}{c}\left(1-\left[1+\left(\frac{n d}{F}\right)^{2}-2 \frac{n d}{F} \sin \theta_{s}\right]^{1 / 2}\right)+t_{0}
$$

where $t_{n}$ is the required delay for the $n$th element, $F$ is the focal length, and $t_{0}$ is a constant to keep the delays positive. The focusing has two limitations as it stands. Firstly, it is only valid for an odd number of elements. However, most phased arrays have an even number of elements. Secondly, the constant $t_{0}$ to keep the delays positive is very clumsy to utilize. In order to overcome these deficiencies, a generalized formula for handling both even and odd number of elements was derived. As shown in Figure 7, the following geometric relationship can be attained:

$$
\begin{gathered}
(F \cos \theta)^{2}+\left\{F \sin \theta-\left[(i-1) d-\frac{n-1}{2} d\right]\right\}^{2} \\
=\left[F-\left(c t_{i}-c t_{1}\right)\right]^{2}
\end{gathered}
$$

for the element $n=1,2, \ldots, N$, where $N$ is the total number of elements. $t_{i}$ is the actuate time of the $i$ th element. Solving for $t_{i}$, we obtain

$$
\begin{aligned}
t_{i}= & \frac{F}{c} \\
& \times\left[1-\sqrt{1+\frac{d^{2}}{F^{2}}\left(i-\frac{n+1}{2}\right)^{2}-\frac{2 d}{F}\left(i-\frac{n+1}{2}\right) \sin \theta}\right] \\
& +t_{1} .
\end{aligned}
$$




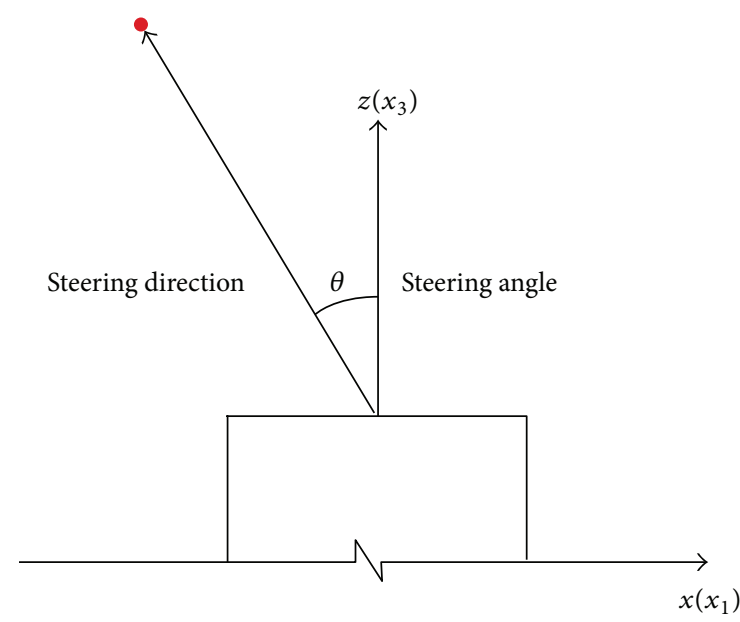

(a)

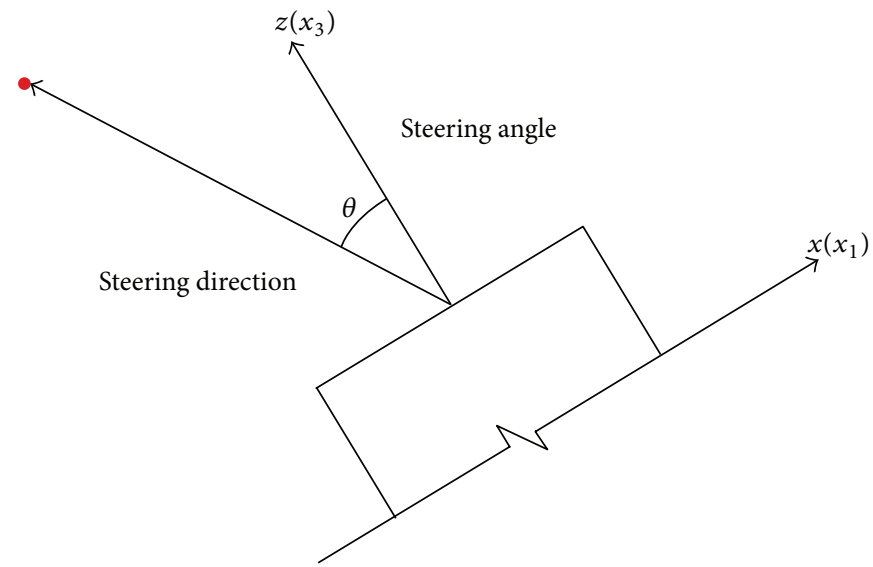

(b)

FIGURE 8: (a) A steering angle of a phased array transducer. (b) A conventional transducer rotated at an angle.

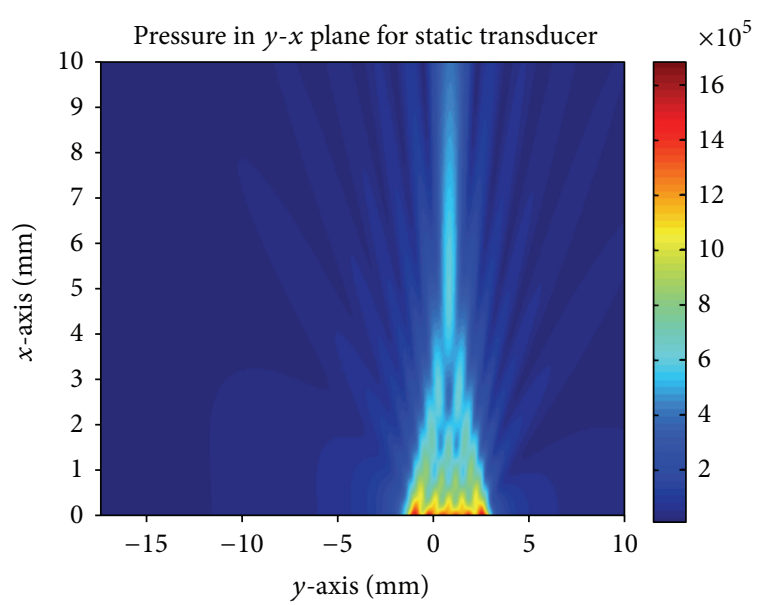

(a)

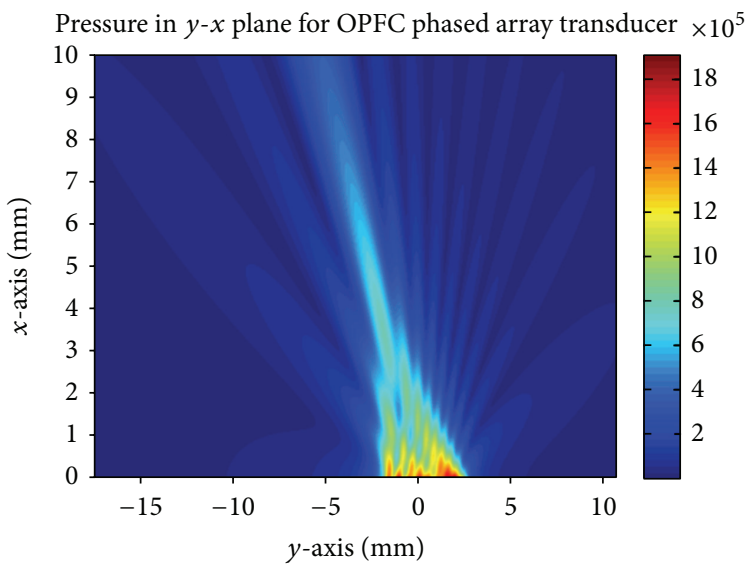

(c)

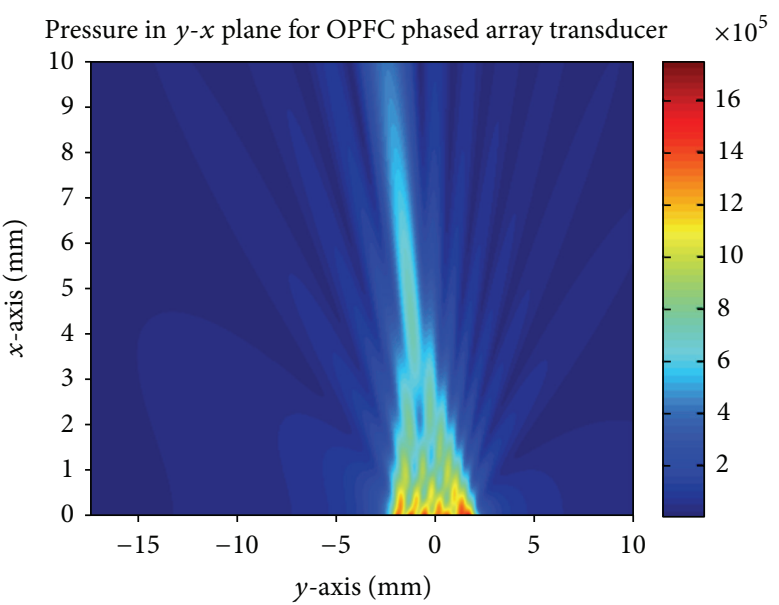

(b)

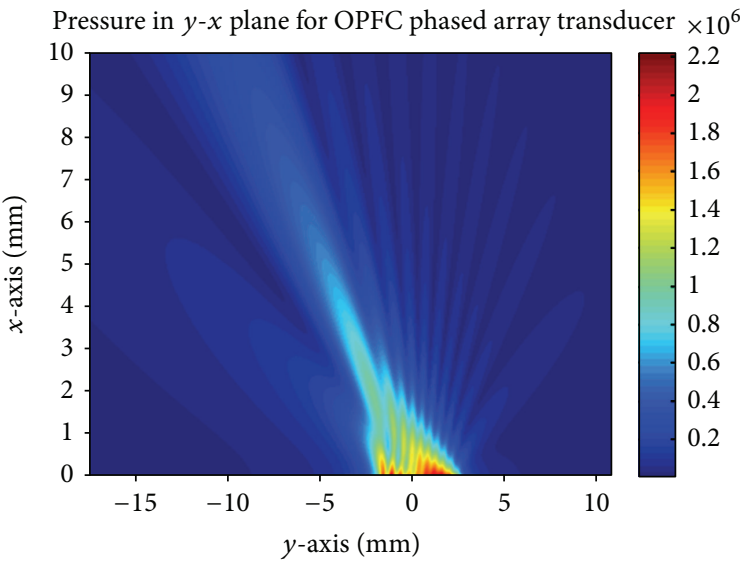

(d)

FIGURE 9: Acoustic pressure field generated by an OPFC phased array transducer for steering angles (a) $0^{\circ}$, (b) $15^{\circ}$, (c) $30^{\circ}$, and (d) $45^{\circ}$. 


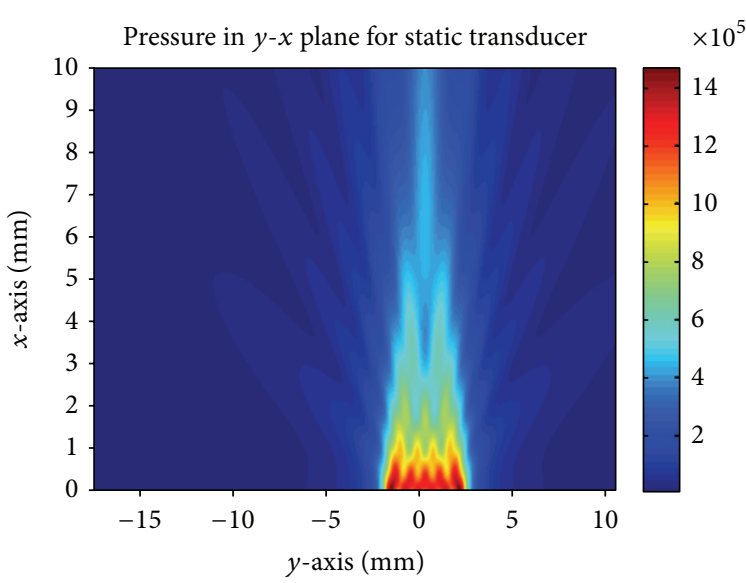

(a)

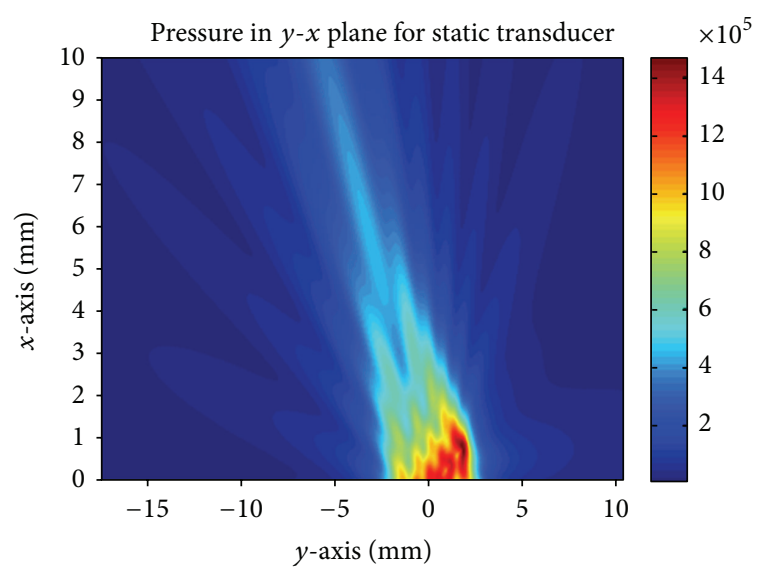

(c)

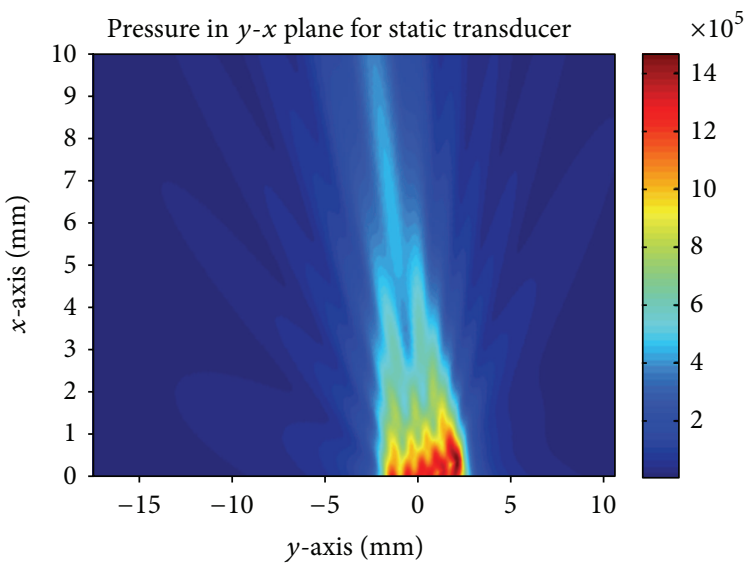

(b)

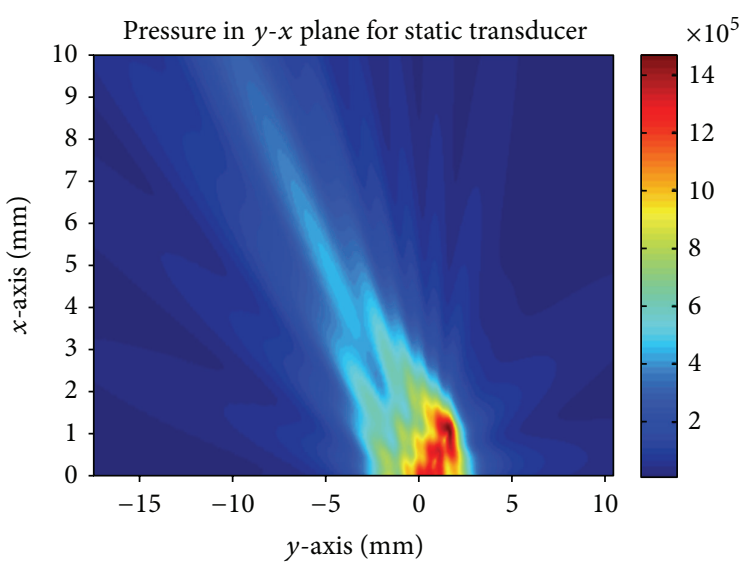

(d)

FIGURE 10: Acoustic pressure field generated by a traditional transducer for steering angles (a) $0^{\circ}$, (b) $15^{\circ}$, (c) $30^{\circ}$, and (d) $45^{\circ}$.

Consider Taylor expression,

$$
\begin{aligned}
{[1} & \left.+\frac{d^{2}}{F^{2}}\left(i-\frac{n+1}{2}\right)^{2}-\frac{2 d}{F}\left(i-\frac{n+1}{2}\right) \sin \theta\right]^{1 / 2} \\
& \approx 1-\frac{d}{F}\left(i-\frac{n+1}{2}\right) \sin \theta+\frac{1}{2}\left[\frac{d}{F}\left(i-\frac{n+1}{2}\right) \cos \theta\right]^{2} .
\end{aligned}
$$

Substituting (20) into (21), the focusing delay of adjacent arrays can be obtained:

$$
\Delta \tau_{i}=t_{i}-t_{i-1}=\frac{d \sin \theta}{c}+\frac{d^{2}}{2 c F}[n-2(i-1)] \cos ^{2} \theta
$$

and $\Delta \tau_{1}=d \sin \theta / c$.

So,

$$
\Delta \tau_{i}=\Delta \tau_{1}+\frac{c \Delta \tau_{1}^{2}}{2 F \tan ^{2} \theta}[n-2(i-1)] .
$$

The delay of the $i$ th element and the first element can be written as

$$
\Delta t_{i}=(i-1) \Delta \tau_{1}+\frac{c \Delta \tau_{1}^{2}}{2 F \tan ^{2} \theta}[n-2(i-1)] .
$$

OPFC phased array transducer is composed of many elements arranged in a certain pattern that emit acoustic energy at different times. The elements can be pulsed in certain sequence to control the beam angle depending on the time delay.

4.2. Numerical Simulation Results and Discussion. OPFC phased array transducer can easily steer an ultrasonic beam in different directions without rotating or moving. But the traditional transducers are rotated to obtain the desired steering direction. Figure 8 shows the steering sketch of phased array transducer and conventional transducer rotated.

Figure 9 shows the acoustic pressure field generated by an OPFC phased array transducer in water. The length and width of the transducer side are $5 \mathrm{~mm}$ and $0.8 \mathrm{~mm}$, respectively. And the exciting frequency is $2 \mathrm{MHz}$. The acoustic pressure fields are modeled for steering angles equal to $0^{\circ}, 15^{\circ}, 30^{\circ}$, and $45^{\circ}$, respectively. Figures $10(\mathrm{a})-10(\mathrm{~d})$ show the pressure field generated by eight single OPFC sensor elements when the axis of the transducer is rotated by $0^{\circ}, 15^{\circ}, 30^{\circ}$, and $45^{\circ}$, respectively. For both of these results, $x$-axis stands for the coordinate in length direction of OPFC phased array transducer and $y$-axis for the coordinate in width direction. 


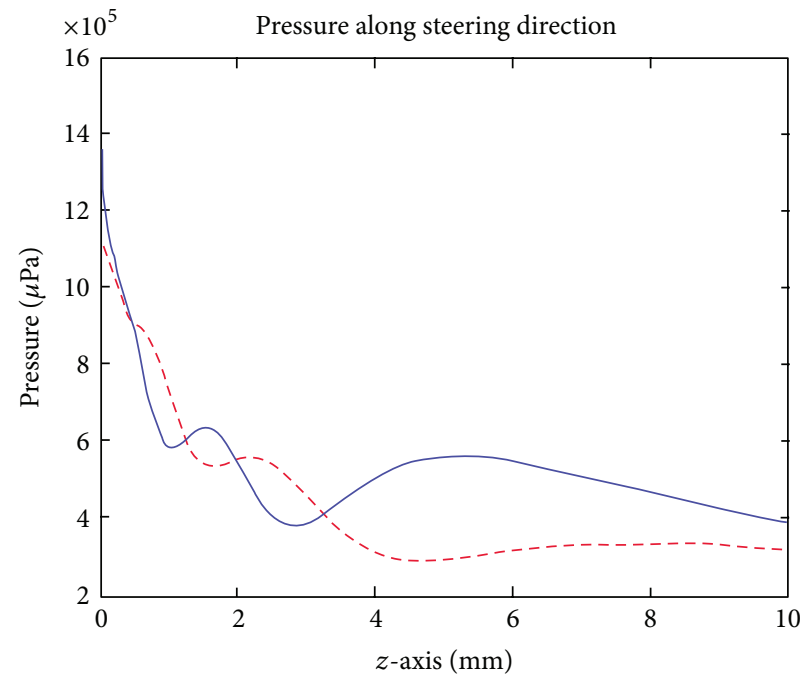

(a)

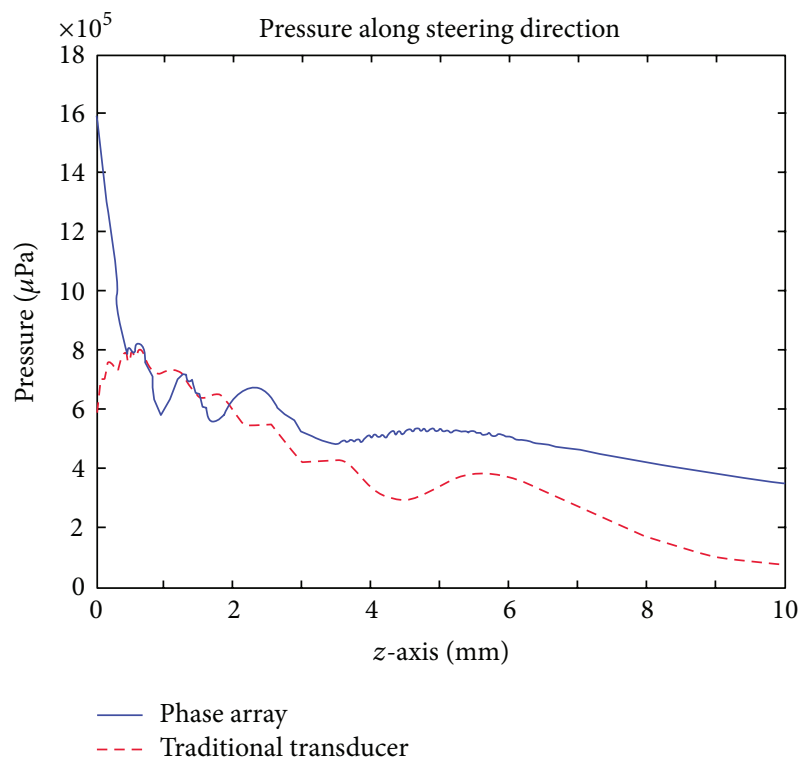

(c)

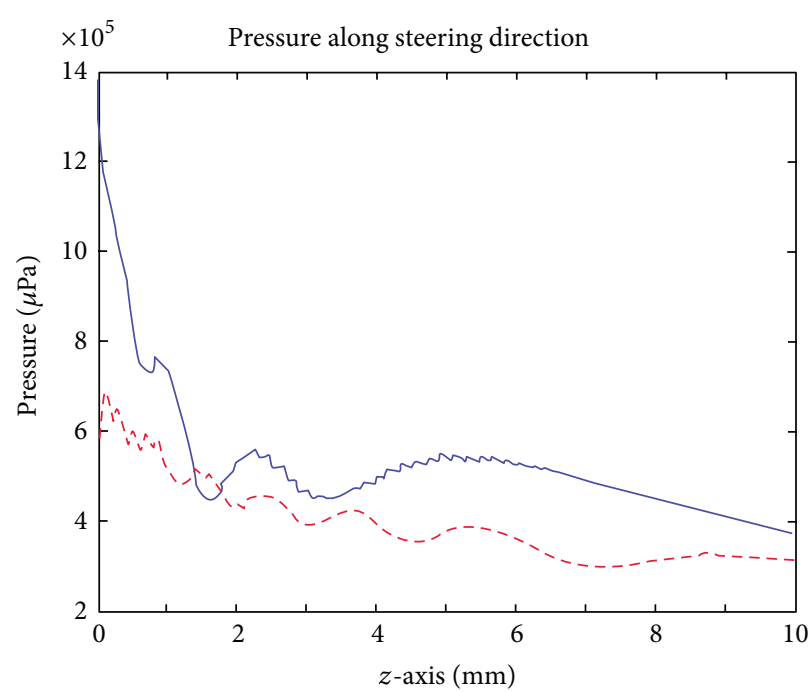

(b)

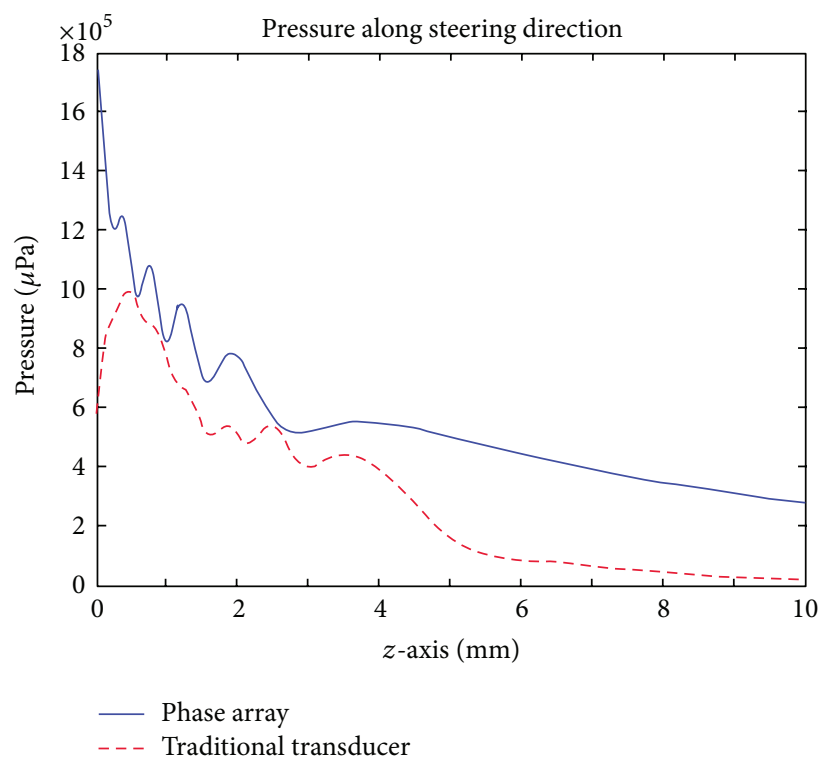

(d)

FIGURE 11: Comparison of the pressure by phased array and traditional transducer along the axis.

The surface area is equal to $4.0 \mathrm{~mm}^{2}$ and the number of point sources is 80 . Each OPFC element is discredited into five point sources distributed along the $z$ direction, giving rise to a total of 60 point sources. In Figure 9, the distance of the focal point is set at $5 \mathrm{~mm}$ in the $x$ direction. The normal distance in $x$ direction kees stable at the location of $5 \mathrm{~mm}$ as the steering angles changes. From Figures 9 and 10, it can be seen that the maximum pressure intensities are $1.6 \times 10^{6}$ and $1.45 \times 10^{6}$ units for phased array and conventional transducers, respectively. It is also observed that the pressure beam produced by the OPFC phased array transducer is narrower or more collimated than that produced by the traditional transducer. Similar conclusions can be drawn from Figures $9(\mathrm{~b})$ and $10(\mathrm{~b})$ for $15^{\circ}$ steering angle, Figures 9 (c) and 10 (c) for $30^{\circ}$ steering angle, and Figures 9 (d) and 10(d) for $45^{\circ}$ steering angle.
Figure 11 is the acoustic pressure along the axis by phased array and traditional transducers for steering angles equal to $0^{\circ}, 15^{\circ}, 30^{\circ}$, and $45^{\circ}$, respectively. From the pictures, the OPFC phased array transducer produces stronger pressure beam than the traditional transducer in the near field along steering direction. The simulation shows that the pressure excited by OPFC phased array transducer is stronger than that by traditional transducer between the near field distance $N_{f}$ and the focal point $(Z=10 \mathrm{~mm})$.

The interaction effect between two OPFC phased array transducers placed in the same medium is studied. Two transducers faces are placed parallel to each other and the central axes are collinear. The acoustic field generated by the one end as well as the scattered fields by the other end transducers is calculated. The surrounding medium is water. 


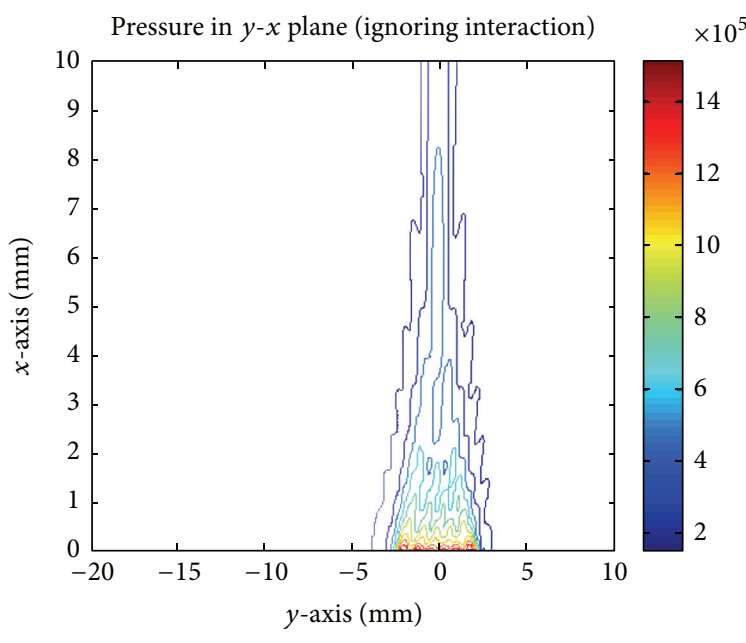

(a)

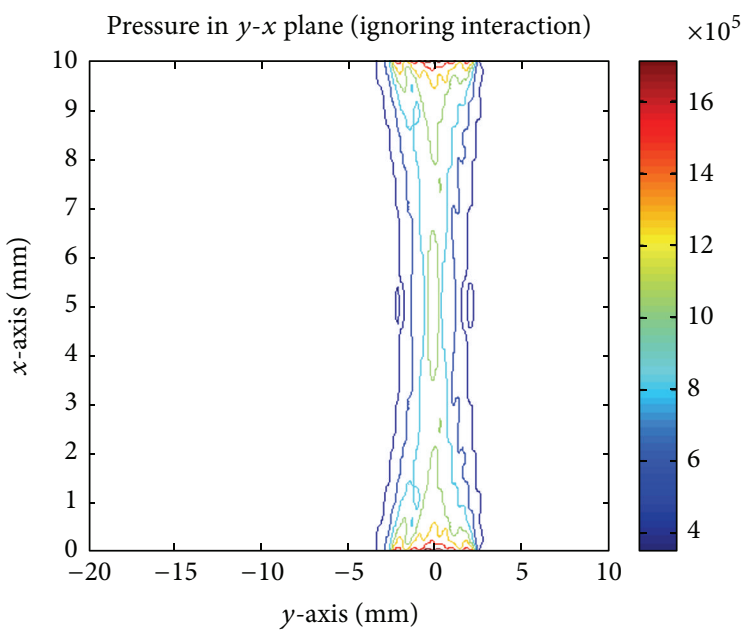

(b)

FIgURE 12: Acoustic pressure in the $x-y$ plane in different interaction effects. (a) Acoustic pressure in the $x-z$ plane for steering angle 0 . (b) Acoustic pressure in the $x-z$ plane when the interaction effect is considered.

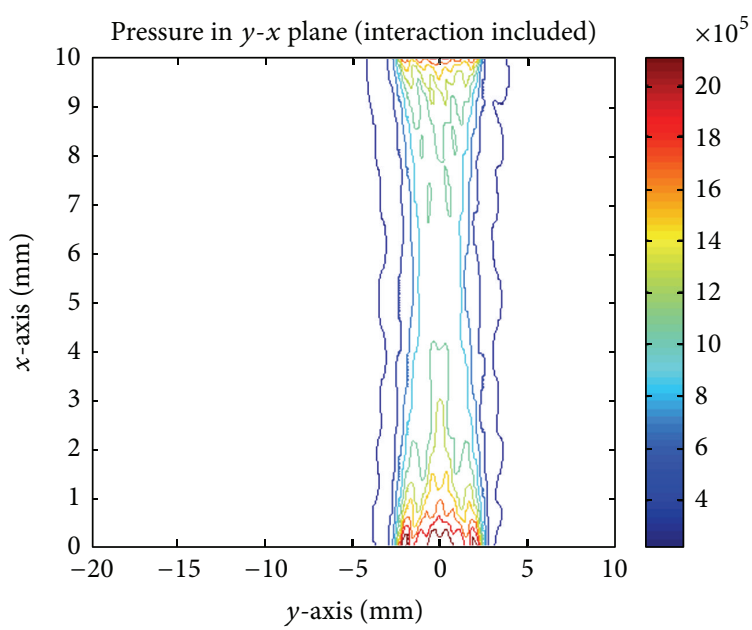

Figure 13: Acoustic pressure in the $x-y$ plane when the interaction effect is included.

Figures 12(a) and 12(b) show the pressure fields generated by the phased array transducers at the steering angle $0^{\circ}$ when the interaction effect is ignored. Figure 13 shows the pressure field by the same OPFC phased array transducers when the interaction effect is included. The pressure beam generated by the bottom transducer is partly reflected or scattered by the top transducer and affects the total acoustic field. The interaction effect can be seen clearly. The reflected beam from the top transducer caused peak value is much greater than that observed in Figure 12. In Figure 13, the beam at near $z=5 \mathrm{~mm}$ is slightly wider than that in Figure 12(b).

\section{Conclusion}

The fabrication principle of OPFC linear phased array is demonstrated for the special orthotropic performance. An OPFC linear phased array transducer with dynamic focusing is modeled by using DPSM. DPSM technology was utilized to compare the behavior of focusing in different angles of
OPFC linear phased array and traditional transducer. Two linear phased array transducers placed face to face in the same medium are also modeled to study modeled to study the interaction effect. This demonstrated the importance of focusing in the near fields, while the directivity for focusing is very well defined. The OPFC linear phased array transducer can produce stronger and better collimated beams to detect the damages.

\section{Conflict of Interests}

The authors declare that there is no conflict of interests regarding the publication of this paper.

\section{Acknowledgments}

The authors thank the support of " 863 Project" (2009AA03Z107), Natural Science Foundation of China 
(11272138), Jiangsu University Foundation (14JDG022), Doctor Point Foundation Project (20123227130002), and their colleagues for their contribution to the work.

\section{References}

[1] J. V. Hatfield, N. R. Scales, A. D. Armitage, P. J. Hicks, Q. X. Chen, and P. A. Payne, "An integrated multi-element array transducer for ultrasound imaging," Sensors and Actuators A: Physical, vol. 41, no. 1-3, pp. 167-173, 1994.

[2] A. Erhard, G. Schenk, T. Hauser, and U. Völz, "New applications using phased array techniques," Nuclear Engineering and Design, vol. 206, no. 2-3, pp. 325-336, 2001.

[3] J.-P. Ardouin and A. N. Venetsanopoulos, "Modelling and restoration of ultrasonic phased-array B-scan images," Ultrasonic Imaging, vol. 7, no. 4, pp. 321-344, 1985.

[4] Y. Luo and Z. Wang, "Fabrication and performance evaluation of OPCM array transducer," Applied Mechanics and Materials, vol. 83, pp. 109-115, 2011.

[5] T. Baoqi, Smart Material and Structure Systems, National Defense Industry Press, Beijing, China, 1997.

[6] Z. Wang, Y. Luo, and W. Sun, "Optimal design of OPCM sensor by the orthotropic ratio," Key Engineering Materials, vol. 464, pp. 350-353, 2011.

[7] Y. Luo, H.-G. Liu, G.-Q. Zhao, and J.-Z. Gu, "Calibration of OPCM sensor for surface elastic waves," Optics and Precision Engineering, vol. 16, no. 12, pp. 2334-2340, 2008.

[8] D. Placko, T. Kundu, and R. Ahmad, "Ultrasonic field computation in the presence of a scatterer of finite dimension," in Smart Nondestructive Evaluation and Health Monitoring of Structural and Biological Systems, vol. 5047 of Proceedings of the SPIE, pp. 169-179, March 2003.

[9] V. Giurgiutiu, Structural Health Monitoring with Piezoelectric Wafer Active Sensors, Academic Press, 2007.

[10] Z. Hai Lan, Theoretical Acoustics, Higher Education Press, 2007.

[11] L. Azar, Y. Shi, and S.-C. Wooh, "Beam focusing behavior of linear phased arrays," NDT and E International, vol. 33, no. 3, pp. 189-198, 2000. 


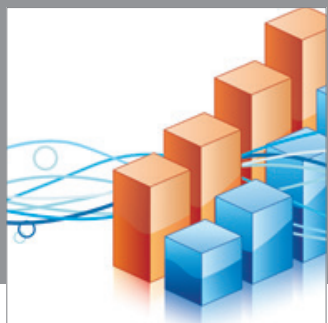

Advances in

Operations Research

mansans

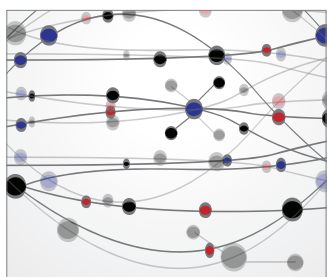

The Scientific World Journal
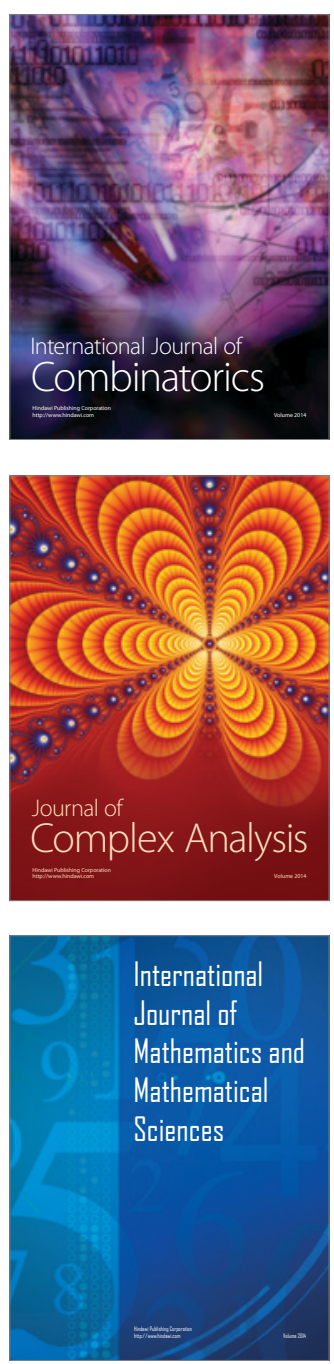
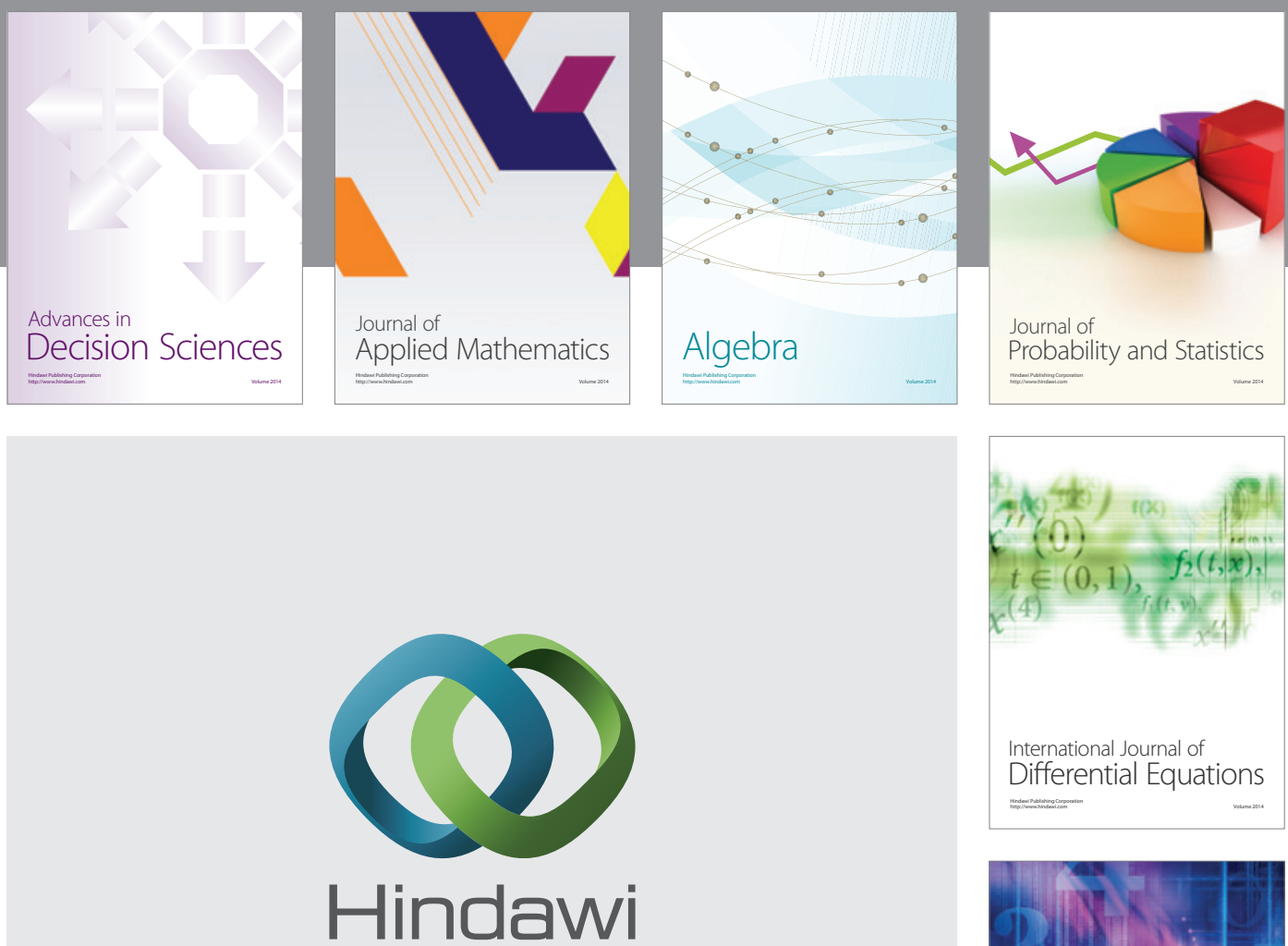

Submit your manuscripts at http://www.hindawi.com
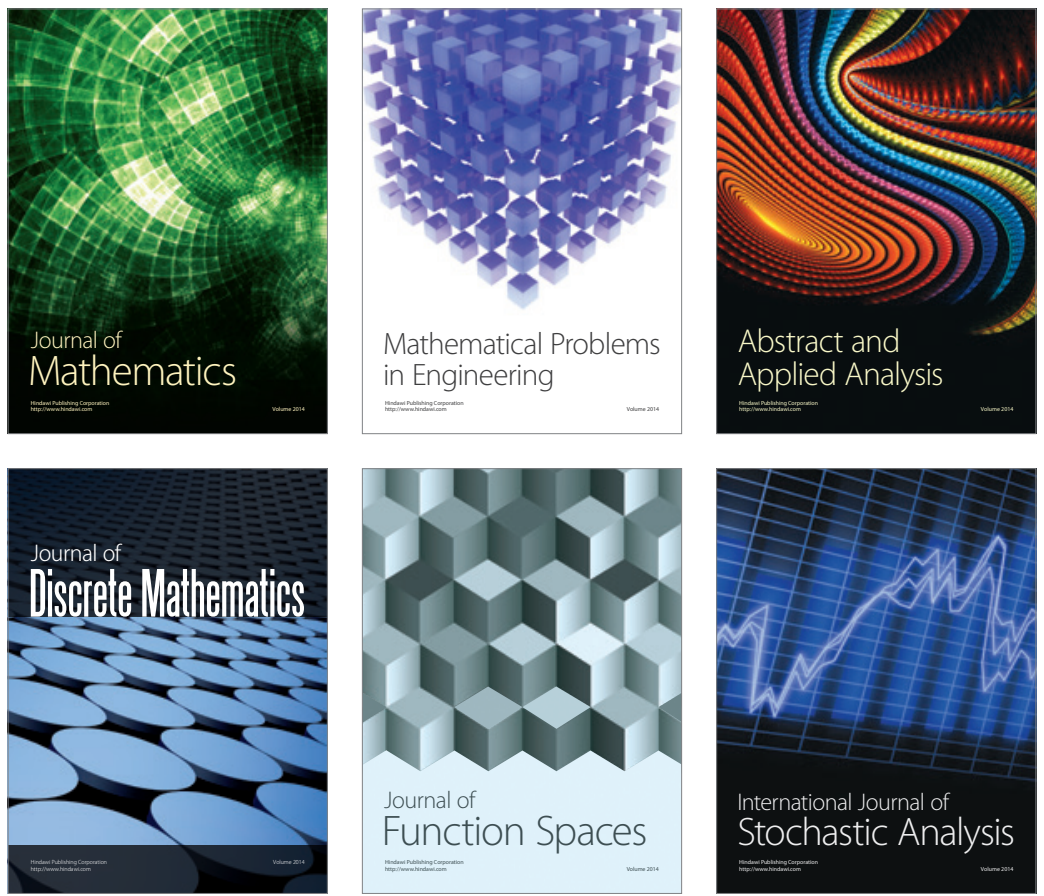

Journal of

Function Spaces

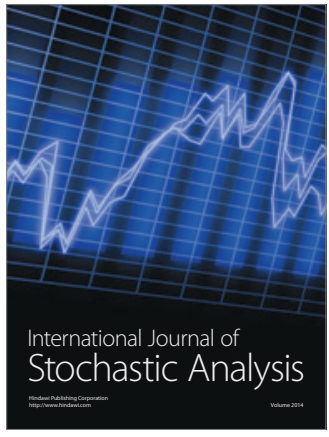

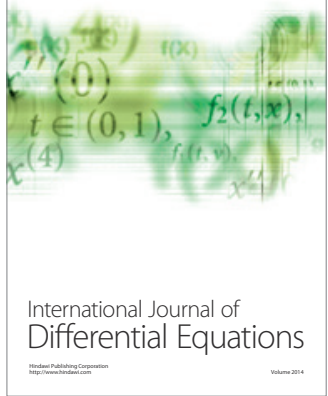
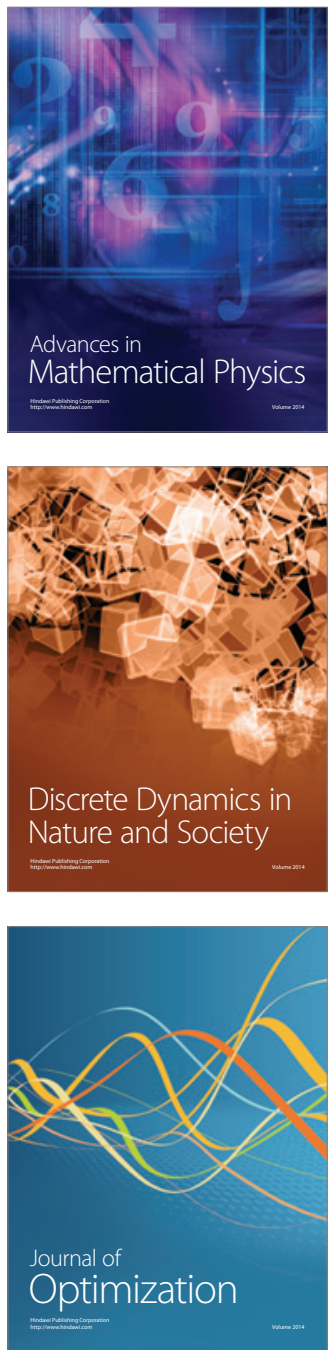